\title{
No association between ApoE polymorphism and febrile seizures
}

\author{
Pierre Lavenex ${ }^{1,2} \cdot$ Pamela Banta Lavenex $^{1} \cdot$ François Cachat $^{3} \cdot$ Mario Gehri $^{4}$ • \\ Typhaine Juvet ${ }^{2}$
}

\begin{abstract}
Seizures associated with fever are a common pediatric problem, affecting about $2-7 \%$ of children between 3 months and 5 years of age. Differentiation of febrile seizures from acute symptomatic seizures secondary to central nervous system infections or seizures associated with fever in children with epilepsy is essential to provide appropriate treatment and follow-up care. Here, we tested the hypothesis that children who exhibit simple febrile seizures during early childhood, but do not develop epileptic seizures later in life, might preferentially carry the ApoE2 allele of the gene coding for the apolipoprotein E. We did not find any differences in the distribution of ApoE alleles or genotypes between individuals who exhibited simple febrile seizures $(n=93)$ and age-matched, typically developing subjects $(n=80)$. We found that the observed allele and genotype frequencies did not deviate from Hardy-Weinberg equilibrium, which suggests that the frequencies of ApoE alleles and genotypes are stable in the Swiss population from which our samples were derived. Across both groups of subjects $(n=173)$, we found an ApoE2 allele frequency of 0.064 , an ApoE3 frequency of
\end{abstract}

$\triangle$ Pierre Lavenex

pierre.lavenex@unil.ch

1 Laboratory for Experimental Research on Behavior, Institute of Psychology, University of Lausanne, 1015 Lausanne, Switzerland

2 Laboratory of Brain and Cognitive Development, Department of Medicine, Fribourg Center for Cognition, University of Fribourg, 1700 Fribourg, Switzerland

3 Department of Pediatrics, Hôpital du Samaritain, 1800 Vevey, Switzerland

4 Hôpital de l'Enfance, Centre Hospitalier Universitaire Vaudois, 1000 Lausanne, Switzerland
0.829 and an ApoE4 frequency of 0.107. Our findings are consistent with previous reports of the distribution of ApoE polymorphism for European subjects free of any neurological disorders, and show that the different alleles of the gene coding for the apolipoprotein E are not associated with the occurrence of simple febrile seizures.

Keywords Genetics - Epilepsy · Hippocampus - Fever . Astrocytes · Prostaglandins - Apolipoprotein .

Development $\cdot$ Children $\cdot$ Pediatrics

\section{Introduction}

Seizures associated with fever are a common pediatric problem, affecting about 2-7\% of children between 3 months and 5 years of age [1]. Although the vast majority of children who present with febrile seizures do not develop epilepsy, the distinction between epilepsy and febrile seizures is important since the first manifestation of the epilepsy syndrome might occur during a fever episode at a young age. If a diagnosis of epilepsy has been established prior to the episode, appropriate therapeutic measures are already in place. If not, however, physicians must wait to see if further seizures develop in order to provide follow-up care. Adequate diagnosis is, therefore, key to adequate treatment and patient education that can alleviate parents' anxiety and allow the family return to normal life. To achieve this goal, a number of researchers and practitioners have called for an increased effort to determine the genetic factors predisposing to febrile seizures and understand the relation between febrile seizures and specific epilepsy syndromes [2-8].

The importance of genetic factors in the incidence of febrile seizures has long been recognized [9, 10], but 
specific genes that affect febrile seizure cases, and which are not part of a broader phenotypic spectrum that includes afebrile seizures, have yet to be conclusively identified [5, 11]. For example, interleukin 1-beta has been linked to the generation of febrile seizures [3], but increased frequency of the interleukin-1-beta-511T allele has also been reported in patients with temporal lobe epilepsy [12]. Moreover, the mechanisms by which variants of these particular genes could contribute specifically to febrile seizures that are not associated with epilepsy have yet to be proposed.

Here, we tested the hypothesis that children who exhibit simple febrile seizures during early childhood, but do not develop epileptic seizures later in life, might preferentially carry the ApoE2 allele of apolipoprotein E. This hypothesis was based on findings showing that a number of genes related to astrocytic processes and functions are differentially expressed in the primate hippocampus across postnatal development [13]. In humans, the highest incidence of seizures is in the first 2 years of life and is most often associated with a febrile illness [14]. Fever begins with the activation of immune response cells that produce interleukin-1, which in turn increases prostaglandin E2 synthesis [15]. Prostaglandins act at the level of the hypothalamus to regulate body temperature and induce fever. Interestingly, prostaglandins also stimulate calciumdependent glutamate release in astrocytes [16, 17], which can induce abnormal prolonged depolarization with repetitive spiking in CA1 pyramidal neurons leading to seizures $[18,19]$. Thus, the relatively high astrocytic coverage of the dense network of CA1 excitatory synapses in the newborn [13] might contribute to a higher incidence of febrile seizures. The decrease in astrocytic coverage of hippocampal excitatory synapses with development might in turn reduce the probability of neuronal depolarization evoked by astrocytic glutamate release, and thus contribute to the decreased susceptibility to febrile seizures with age. The pertinent question, then, is why only $2-7 \%$ of children exhibit febrile seizures [2, 3, 6-8]?

To address this question, we considered the molecular machinery involved in glutamate uptake and release in astrocytes. We focused on ApoE as a candidate gene for three reasons: (1) ApoE is located on chromosome $19 q$ thought to contain susceptibility genes associated with febrile seizures, (2) three different genotypes (ApoE2, ApoE3, ApoE4), linked to single nucleotide polymorphisms, have been identified in humans, and (3) apolipoprotein $\mathrm{E}$ found in the brain is synthesized in astrocytes and plays a major role in the maintenance of lipid homeostasis and vesicular trafficking [20, 21]. In Europeans free of any neurological disorders, the distribution of different alleles is stable, with an ApoE2 frequency of 0.068, an ApoE3 frequency of 0.813 and an ApoE4 frequency of 0.119 [22]. ApoE2 is more highly retained in astrocytes than ApoE3 and ApoE4 (Fan and Fazio, personal communication; see [23] for similar results in macrophages) and might thus facilitate the prostaglandin-induced, calcium-dependent exocytosis of glutamate by astrocytes $[16,17]$. In turn, increased glutamate concentration in the synaptic cleft might induce prolonged depolarization with repetitive spiking in neurons leading to seizures. Interestingly, previous studies have considered the potential link between ApoE and seizures, with some studies specifically interested in febrile seizures focusing their analyses on ApoE4 [24], the allele known to be a risk factor for Alzheimer's disease, and other studies considering all three ApoE alleles included other seizure phenotypes [25, 26]. However, none of the previous studies was adequately designed to test the hypothesis that ApoE2 might confer a specific predisposition to febrile seizures.

\section{Materials and methods}

We recruited ninety-three children (45 females, 48 males), previously diagnosed with a febrile seizure in two hospitals of the Lemanic Region, Canton of Vaud, Switzerland: the Hôpital du Samaritain, Department of Pediatrics, in Vevey and the Hôpital de l'Enfance, Centre Hospitalier Universitaire Vaudois, in Lausanne. Selection criteria included a documented history of simple febrile seizure (FS), the absence of any other neurological disorders (in particular other seizure disorders), and the absence of a family history of neurological or neuropsychiatric disorders. The characteristics of the febrile seizure and the family history of each patient were evaluated in their personal hospital records, and again by a senior pediatrician (F.C. or M.G.) during the encounter with the family. Patients with unclear history were not enrolled. FS patients experienced a single febrile seizure episode at an average age of 1.8 years (standard deviation 1.0 years, median age 1.5 years) and were enrolled in the study at an average age of 9.5 years (standard deviation 3.1 years, median age 9.7 years). Eighty age-matched typically developing (TD) controls (28 females, 52 males) were also recruited at an average age of 9.5 years (standard deviation 2.5 years, median age 9.5 years), via the same recruitment system. The absence of FS in the patient or his/her family or any other seizure history was also ascertained by a senior pediatrician, at the time of saliva sample collection.

FS and TD subjects were asked to provide a saliva sample to obtain individual samples of genomic DNA. Saliva was collected with the OG-500 Oragene-DNA selfcollection kit (DNAgenothek, Kanata, ON, Canada), and returned to our laboratory by the participants via priority mail. Upon receipt, samples were anonymized and heated at $50^{\circ}$ for $1 \mathrm{~h}$ to ensure that DNA was adequately released 
and that nucleases were permanently deactivated, following the manufacturer's protocol (DNAgenothek, Kanata, ON, Canada). DNA extraction and ApoE analyses were performed following the protocols provided with the commercially available ApoE StripAssay ${ }^{\mathrm{TM}}$ for the identification of apolipoprotein E isoforms E2, E3 and E4 based on polymerase chain reaction (PCR) and reverse hybridization (ViennaLab Labiordiagnostika $\mathrm{GmbH}$, Vienna, Austria). ApoE has three common alleles (E2, E3, and E4), differing by the presence of either the $\mathrm{C}$ or $\mathrm{T}$ nucleotide at codons 112 and 158 in the fourth exon of the ApoE gene. ApoE2 contains the sequence TGC coding for cysteine at both positions, ApoE3 contains the sequence TGC at codon 112 (coding for cysteine) and the sequence CGC at codon 158 (coding for arginine), and ApoE4 contains the sequence CGC at both codons, therefore, coding for arginine at both positions. The six possible homozygous and heterozygous ApoE genotypes (E2/E2, $\mathrm{E} 2 / \mathrm{E} 3, \mathrm{E} 2 / \mathrm{E} 4, \mathrm{E} 3 / \mathrm{E} 3, \mathrm{E} 3 / \mathrm{E} 4, \mathrm{E} 4 / \mathrm{E} 4)$ result in a combination of the respective individual isoforms that can easily be visualized with the ApoE StripAssay. The distribution of allele frequencies (ApoE2, ApoE3, ApoE4) was calculated from the observed genotype distributions. Odds ratios were calculated as $(\mathrm{p} 1 \times(1-\mathrm{p} 1)) /(\mathrm{p} 2(1-\mathrm{p} 2))$, with $\mathrm{p} 1$ corresponding to the probability of the allele in the population of subjects with febrile seizures and p2 corresponding to the probability of the allele in the population of control subjects. Ninety-five percent confidence intervals (95\% CI) of odds ratios were calculated using the following formula $\left(\mathrm{e}\left(\ln (\mathrm{OR}) \pm \operatorname{sum}(1 / x)^{0.5}\right)\right.$. Chi square statistical tests were performed to compare the number of individual genotypes and allele distributions in both populations. Statistical significance was set at $p<0.05$.

This work was approved by the Cantonal Ethics Commission (Vaud) for Human Research (protocol no. 33/10), and conducted in accordance with the Declaration of Helsinki. The parents of all participants gave informed written consent.

\section{Results}

\section{Alleles distribution}

We did not find any differences in the distribution of ApoE alleles between FS and TD subjects $\left(\chi^{2}=1.095, d f=2\right.$, $p=0.5783$; Table 1). Across the two groups of subjects recruited in the Lemanic region of Switzerland, we found an ApoE2 allele frequency of 0.064, an ApoE3 frequency of 0.829 and an ApoE4 frequency of 0.107. These findings are consistent with previous findings including 24,262 European subjects free of any neurological disorders, for which the distribution of the different alleles was rather
Table 1 Distribution of ApoE alleles in individuals who exhibited febrile seizures (FS) and age-matched, typically developing subjects (TD)

\begin{tabular}{llll}
\hline \multicolumn{1}{l}{ E2 } & E3 & E4 \\
\hline Observed numbers & & & \\
FS $(n=93)$ & 10 & 154 & 22 \\
TD $(n=80)$ & 12 & 133 & 15 \\
Total $(n=173)$ & 22 & 287 & 37 \\
Chi square & $p=0.4347$ & $p=0.9747$ & $p=0.4866$ \\
Observed frequencies & & & \\
FS & 0.0538 & 0.8280 & 0.1183 \\
TD & 0.0750 & 0.8313 & 0.0938 \\
Total & 0.0636 & 0.8295 & 0.1069 \\
Odds ratio & 0.733 & 1.015 & 1.227 \\
95 \% CI OR & $0.420-1.279$ & $0.582-1.771$ & $0.704-2.141$ \\
\hline
\end{tabular}

stable, with an ApoE2 allele frequency of 0.068, an ApoE3 frequency of 0.813 and an ApoE4 frequency of 0.119 (computed from data compiled by [22]).

\section{Genotypes distribution}

We did not find any differences in the distribution of ApoE genotypes between FS and TD subjects $\left(\chi^{2}=4.842\right.$, $d f=5, p=0.4354$; Table 2). Across the two groups of subjects recruited in the Lemanic region of Switzerland, we found that the most prevalent genotype was E3-E3 with $70.5 \%$ of subjects, followed by E3-E4 with $16.2 \%$ and E2-E3 with $8.7 \%$; the other three genotypes (E2-E2, E2E4, E4-E4) accounted for less than $5 \%$ of the sampled population.

\section{Hardy-Weinberg equilibrium}

We compared the observed frequencies of ApoE genotypes (Table 2) with the expected frequencies based on the Hardy-Weinberg principle $\left((\text { ApoE2 }+ \text { ApoE3 }+ \text { ApoE4 })^{2}=1\right.$; Table 3) and found that the genotype frequencies did not differ from equilibrium $\left(\mathrm{FS}: \quad \chi^{2}=4.338, \quad d f=5, \quad p=0.5018 ; \quad \mathrm{TD}\right.$ : $\chi^{2}=2.461, d f=5, p=0.7823$; total: $\chi^{2}=6.035, d f=5$, $p=0.3028$ ). This suggests that the frequencies of ApoE alleles and genotypes are stable in the Swiss population from which our samples were derived.

\section{Discussion}

We hypothesized that children who exhibit febrile seizures during early childhood, but do not develop epileptic seizures later in life, might preferentially carry the ApoE2 
Table 2 Distribution of ApoE genotypes in individuals who exhibited febrile seizures (FS) and age-matched, typically developing subjects (TD)
Table 3 Predicted distribution of ApoE genotypes in individuals who exhibited febrile seizures (FS) and agematched, typically developing subjects (TD), following the Hardy-Weinberg equation for equilibrium

\begin{tabular}{lllllll}
\hline & E2-E2 & E2-E3 & E2-E4 & E3-E3 & E3-E4 & E4-E4 \\
\hline FS $(n=93)$ & 0 & 7 & 3 & 66 & 15 & 2 \\
TD $(n=80)$ & 1 & 8 & 2 & 56 & 13 & 0 \\
Total $(n=173)$ & 1 & 15 & 5 & 122 & 28 & 2 \\
Chi square & $p=0.3346$ & $p=0.7074$ & $p=0.6449$ & $p=0.2362$ & $p=0.5967$ & $p=0.1713$ \\
Frequencies & & & & & & \\
FS & 0.0000 & 0.0753 & 0.0323 & 0.7097 & 0.1613 & 0.0215 \\
TD & 0.0125 & 0.1000 & 0.0250 & 0.7000 & 0.1625 & 0.0000 \\
Total & 0.0058 & 0.0867 & 0.0289 & 0.7052 & 0.1618 & 0.0116 \\
\hline
\end{tabular}

\begin{tabular}{lllllll}
\hline & E2-E2 & E2-E3 & E2-E4 & E3-E3 & E3-E4 & E4-E4 \\
\hline Numbers & & & & & & \\
$\quad$ FS $(n=93)$ & 0 & 8 & 1 & 63 & 18 & 1 \\
TD $(n=80)$ & 0 & 10 & 1 & 56 & 13 & 1 \\
$\quad$ Total $(n=173)$ & 1 & 17 & 2 & 108 & 28 & 2 \\
Frequencies & & & & & & \\
FS & 0.0029 & 0.0890 & 0.0127 & 0.6855 & 0.1959 & 0.0140 \\
TD & 0.0056 & 0.1247 & 0.0141 & 0.6910 & 0.1559 & 0.0088 \\
Total & 0.0040 & 0.1055 & 0.0136 & 0.6880 & 0.1774 & 0.0114 \\
\hline
\end{tabular}

The genotype frequencies of ApoE were in Hardy-Weinberg equilibrium for both groups allele of apolipoprotein E. In contrast, we did not find any differences in the frequencies of ApoE alleles or genotypes between FS and TD individuals. We found that the observed allele and genotype frequencies did not deviate from Hardy-Weinberg equilibrium. Across both groups ( $n=173$ subjects), we found an ApoE2 allele frequency of 0.064, an ApoE3 frequency of 0.829 and an ApoE4 frequency of 0.107 . Our findings are thus consistent with previous reports for European subjects free of any neurological disorders, in particular with previous results for a population of 173 subjects derived from the same Lemanic region of Switzerland (Geneva; [27]): ApoE2 allele frequency of 0.072, ApoE3 frequency of 0.821 and ApoE4 frequency of 0.107 . Our findings indicate that the different alleles of the ApoE gene coding for the apolipoprotein $\mathrm{E}$ are not associated with a predisposition to exhibit febrile seizures during early childhood. One possible caveat of our study is the fact that we analyzed fewer than 100 subjects per group. However, in order to be clinically relevant, any association between one gene's polymorphism and a medical condition should be significant even with such a small number of individuals. If larger numbers of subjects are required to demonstrate a statistically significant association, such information is unlikely to be useful for everyday practice.

A previous study by Giray et al. [24] also failed to find differences in allele and genotype frequencies between 69 patients with febrile convulsions and 75 control subjects derived from the Turkish population. However, the distribution of ApoE alleles in their control group differed from what was previously reported by Corbo et al. [27] based on the genotyping of 8453 individuals $\left(\chi^{2}=21.97, d f=2\right.$, $p<0.0001)$. The frequency of the ApoE2 allele in their control population also differed from that observed in our control population $\left(\chi^{2}=4.21, d f=1, p=0.0401\right)$, raising some questions regarding the reliability of their sampling or their sequencing methods. The attempt by Giray et al. [24] to further define different genetic predispositions between simple and complex febrile seizures is also difficult to justify given the small number of subjects with complex seizures comprised in their study $(n=17)$. Other studies have failed to find a causal relationship between ApoE polymorphism and epilepsy: Blumcke et al. [25] failed to demonstrate an association between ApoE polymorphism and early onset temporal lobe epilepsy; Yeni et al. failed to demonstrate an association between ApoE polymorphism and mesial temporal lobe epilepsy with hippocampal sclerosis; Fu et al. [28] reported that ApoE4 does not increase the risk for temporal lobe epilepsy in the Chinese Han population, in the absence of prior trauma. In contrast, Kauffman et al. [29] reported that the ApoE4 allele might be associated with an earlier onset of temporal lobe epilepsy. Thus, in contrast to the well-established association between ApoE polymorphism and the risk of developing Alzheimer's disease [30], there does not seem to be a simple, direct association between ApoE 
polymorphism and the susceptibility to exhibit seizures of various etiologies.

In sum, although our hypothesis that ApoE2 might confer a predisposition to simple febrile seizures was not validated, it remains possible that the cellular pathways involved in the regulation of glutamate uptake and release by astrocytes are associated with the susceptibility to exhibit febrile seizures early in life. Since hundreds of genes have the potential to influence these cellular pathways, this hypothesis could be further tested with a genome-wide approach to identify possible genetic markers of febrile seizures. Using this approach, recent studies found that variants of the sodium channel gene SCN1A are associated with general and MMR vaccine-related febrile seizures [4], but also with mesial temporal lobe epilepsy with hippocampal sclerosis, with and without a history of febrile seizures [5]. If such markers can be found, the development and use of cost-effective sequencing technologies available to clinicians should be combined with the development and use of functional analysis tools that can comprehend multiple gene pathways for routine diagnostic. Altogether, such methods might help to better distinguish a benign childhood disorder with an excellent prognosis from other seizure phenotypes associated with an increased risk of developing epilepsy later in life.

Acknowledgments This research was supported by a grant from the Swiss League Against Epilepsy to PL and funds from the University of Fribourg, Switzerland. The Swiss League Against Epilepsy had no involvement in the study design, nor in the collection, analysis or interpretation of the data, nor in the writing of this report or the decision to submit it for publication. We wish to thank all participating children and their families for their invaluable contribution to this project.

\section{Compliance with ethical standards}

Conflict of interest The authors declare that they have no conflict of interest.

\section{References}

1. Annegers JF, Hauser WA, Shirts SB, Kurland LT (1987) Factors prognostic of unprovoked seizures after febrile convulsions. N Engl J Med 316:493-498

2. Baram TZ (2002) Animal models for febrile seizures. In: Baram TZ, Shinnar S (eds) febrile seizures. Academic Press, San Diego, pp 189-198

3. Dube CM, Brewster AL, Baram TZ (2009) Febrile seizures: mechanisms and relationship to epilepsy. Brain Dev 31:366-371

4. Feenstra B, Pasternak B, Geller F, Carstensen L, Wang T, Huang F et al (2014) Common variants associated with general and MMR vaccine-related febrile seizures. Nat Genet 46:1274-1282

5. Kasperaviciute D, Catarino CB, Matarin M, Leu C, Novy J, Tostevin A et al (2013) Epilepsy, hippocampal sclerosis and febrile seizures linked by common genetic variation around SCN1A. Brain 136:3140-3150
6. Leung AKC, Robson WLM (2007) Febrile seizures. J Pediatr Health Care 21:250-255

7. Østergaard JR (2008) Febrile seizures. Acta Paediatr 98:771-773

8. Sadleir LG, Scheffer IE (2007) Febrile seizures. BMJ 334:307-311

9. Greenberg DA, Holmes GL (2002) The genetics of febrile seizures. Febrile seizures. Academic Press, San Diego, pp 249-262

10. Shinnar S (2002) Human data: what do we know about febrile seizures and what further information is needed. In: Baram TZ, Shinnar S (eds) Febrile seizures. Academic Press, San Diego, pp 317-323

11. Deprez L, Jansen A, De Jonghe P (2009) Genetics of epilepsy syndromes starting in the first year of life. Neurology 72:273-281

12. Kanemoto K, Kawasaki J, Yuasa S, Kumaki T, Tomohiro O, Kaji $\mathrm{R}$ et al (2003) Increased frequency of interleukin-1beta-511T allele in patients with temporal lobe epilepsy, hippocampal sclerosis, and prolonged febrile convulsion. Epilepsia 44:796-799

13. Lavenex P, Sugden SG, Davis RR, Gregg JP, Banta Lavenex P (2011) Developmental regulation of gene expression and astrocytic processes may explain selective hippocampal vulnerability. Hippocampus 21:142-149

14. Hauser WA (1994) The prevalence and incidence of convulsive disorders in children. Epilepsia 35(Suppl 2):S1-6

15. Biddle C (2006) The neurobiology of the human febrile response. AANA J 74:145-150

16. Bezzi P, Carmignoto G, Pasti L, Vesce S, Rossi D, Rizzini BL et al (1998) Prostaglandins stimulate calcium-dependent glutamate release in astrocytes. Nature 391:281-285

17. Volterra A, Meldolesi J (2005) Astrocytes, from brain glue to communication elements: the revolution continues. Nat Rev Neurosci 6:626-640

18. Kang N, Xu J, Xu Q, Nedergaard M, Kang J (2005) Astrocytic glutamate release-induced transient depolarization and epileptiform discharges in hippocampal CA1 pyramidal neurons. J Neurophysiol 94:4121-4130

19. Tian GF, Azmi H, Takano T, Xu Q, Peng W, Lin J et al (2005) An astrocytic basis of epilepsy. Nat Med 11:973-981

20. Fagan AM, Holtzman DM, Munson G, Mathur T, Schneider D, Chang LK et al (1999) Unique lipoproteins secreted by primary astrocytes from wild type, apoE $(-/-)$, and human apoE transgenic mice. J Biol Chem 274:30001-30007

21. Gong JS, Kobayashi M, Hayashi H, Zou K, Sawamura N, Fujita $\mathrm{SC}$ et al (2002) Apolipoprotein E (ApoE) isoform-dependent lipid release from astrocytes prepared from human ApoE3 and ApoE4 knock-in mice. J Biol Chem 277:29919-29926

22. Corbo RM, Scacchi R (1999) Apolipoprotein E (APOE) allele distribution in the world. Is APOE*4 a 'thrifty' allele? Ann Hum Genet 63:301-310

23. Fan D, Qiu S, Overton CD, Yancey PG, Swift LL, Jerome WG et al (2007) Impaired secretion of apolipoprotein E2 from macrophages. J Biol Chem 282:13746-13753

24. Giray O, Ulgenalp A, Bora E, Uran N, Yilmaz E, Unalp A et al (2008) Role of apolipoprotein E in febrile convulsion. Pediatr Neurol 39:241-244

25. Blumcke I, Brockhaus A, Scheiwe C, Rollbrocker B, Wolf HK, Elger CE et al (1997) The apolipoprotein E epsilon 4 allele is not associated with early onset temporal lobe epilepsy. NeuroReport 8:1235-1237

26. Yeni SN, Ozkara C, Buyru N, Baykara O, Hanoglu L, Karaagac $\mathrm{N}$ et al (2005) Association between APOE polymorphisms and mesial temporal lobe epilepsy with hippocampal sclerosis. Eur J Neurol 12:103-107

27. Corbo RM, Scacchi R, Mureddu L, Mulas G, Alfano G (1995) Apolipoprotein $\mathrm{E}$ polymorphism in Italy investigated in native plasma by a simple polyacrylamide gel isoelectric focusing 
technique. Comparison with frequency data of other European populations. Ann Hum Genet 59:197-209

28. Fu YH, Lv RJ, Jin LR, Lu Q, Shao XQ, He JS et al (2010) Association of apolipoprotein E polymorphisms with temporal lobe epilepsy in a Chinese Han population. Epilepsy Res 91:253-259

29. Kauffman MA, Consalvo D, Moron DG, Lereis VP, Kochen S (2010) ApoE epsilon4 genotype and the age at onset of temporal lobe epilepsy: a case-control study and meta-analysis. Epilepsy Res 90:234-239

30. Liu CC, Kanekiyo T, Xu H, Bu G (2013) Apolipoprotein E and Alzheimer disease: risk, mechanisms and therapy. Nat Rev Neurol 9:106-118 